\title{
Pensamento Computacional como intervenção para desenvolvimento cognitivo em idosos
}

\author{
Daniel Araújo de Lucena, Isabel Dillmann Nunes
}

\author{
Instituto Metrópole Digital - Universidade Federal do Rio Grande do Norte \\ Natal - RN - Brasil \\ daniel_computehotmail.com, beleimd.ufrn.br
}

\begin{abstract}
Resumo. Ao longo do envelhecimento pode ocorrer uma redução do desempenho cognitivo, comprometendo, por exemplo, as funções executivas. $\mathrm{Na}$ vida diária, os danos às funções executivas resultam em dificuldades no desempenho de atividades comuns como dirigir um carro e no manejo financeiro. Além disso, compromete-se mecanismos relacionadas a resolução de problemas, que é uma das habilidades mais demandadas pelo mercado de trabalho. Isto posto, o objetivo deste trabalho é estimular a cognição e o desenvolvimento da capacidade de resolução de problemas em idosos utilizando o Pensamento Computacional através de atividades desplugadas.
\end{abstract}

\section{Introdução}

Pesquisas apontam para o aumento considerável da população idosa no Brasil e no mundo (ONU, 2014; IBGE, 2018). Para esta parcela da população é comum alterações neurocognitivas nas funções executivas (CARDOSO; LANDENBERGER; ARGIMON, 2017), definidas como habilidades necessárias para lidar com situações flutuantes e ambíguas do relacionamento social e para uma conduta apropriada, responsável e efetiva (ARGIMON et al., 2006), a exemplo da seletividade de estímulos, capacidade de abstração, autocontrole, planejamento e concentração.

Danos relacionados às funções executivas tem efeito negativo na capacidade de planejamento de ações para alcançar metas, ou seja, compromete-se mecanismos relacionados à resolução de problemas (USTÁRROZ; CÉSPEDES, 2005), até mesmo aqueles mais simples, como dirigir um carro e administrar seu próprio dinheiro, por exemplo.

É necessário destacar que as funções executivas são processos cognitivos especializados que auxiliam no planejamento, raciocínio e resolução de problemas (COLLINS; KOECHLIN, 2012). A competência de resolver problemas é listado pelo relatório do Fórum Econômico Mundial como a principal demandada pelo mercado de trabalho. Cerca de $36 \%$ das atividades em todos os setores da economia exigem a habilidade de solucionar problemas (PATI, 2016).

Se o declínio cognitivo, otimizado sobretudo na senescência, é capaz de comprometer a capacidade de resolução de problemas, isso impacta negativamente na possibilidade do idoso permanecer empregado, indo na contramão do atual cenário brasileiro onde essa faixa da população vem adiando a saída do mercado de trabalho, passando de 6,3\% em 2012 para 7,8\% em 2018, segundo o Instituto de Pesquisa Econômica Aplicada (IPEA, 2018).

Evidências têm mostrado que pelo menos parte da perda de funções no envelhecimento pode ser reversível, por meio de técnicas de intervenção como o treino cognitivo, que é capaz de induzir a plasticidade existente no sistema nervoso e, consequentemente, produzir um impacto positivo na qualidade de vida dos idosos (SCHAIE; WILLIS, 1986).

Se o problema do declínio podemos combater através do treino cognitivo (CAVALLI 
et al., 2017), a habilidade de resolução de problemas no idoso pode ser fomentada através do Pensamento Computacional, que a partir dos seus quatro pilares (decomposição, reconhecimento de padrões, abstração e algoritmo), é uma metodologia de resolução de problemas (WING, 2006).

Diante disso, coloca-se o problema de pesquisa deste trabalho: é possível realizar a estimulação cognitiva em idosos por meio do Pensamento Computacional, o qual busca desenvolver a capacidade de resolução de problemas, tão importante ao mercado de trabalho?

Isto posto, o objetivo deste trabalho é estimular a cognição e o desenvolvimento da capacidade de resolução de problemas em idosos utilizando o Pensamento Computacional através de atividades desplugadas.

\section{Metodologia}

Quanto à forma de abordagem, esta pesquisa é categorizada como quali-quanti, pois utiliza elementos qualitativos e quantitativos. Qualitativa, por permitir o contato entre pesquisador e o grupo de idosos (objeto de estudo), possibilitando o conhecimento de suas realidades. Quantitativa por utilizar procedimentos estruturados e instrumentos formais para coleta de dados, analisando dados numéricos através de procedimentos estatísticos (GERHARDT; SILVEIRA, 2009).

Entende-se que sua natureza é aplicada, com objetivos exploratórios, apoiado no levantamento bibliográfico. Já em relação aos procedimentos, optou-se por uma abordagem quase-experimental, pois caracteriza-se por sua execução com grupos de comparação (experimental e controle), não necessitar longos períodos de observação e tomada de dados e pelo fato da pesquisa não permitir uma seleção aleatória da amostra (SHADISH et al., 2001).

\subsection{Participantes}

Esta pesquisa será realizada com idosos que participam dos cursos já existentes para essa faixa etária no Instituto Metrópole Digital. O recrutamento terá como objetivo selecionar 40 pessoas a partir de 60 anos, de ambos os sexos e sem diagnóstico de demência para compor dois grupos de 20 participantes cada: grupo de controle e o grupo de pesquisa.

\subsection{Instrumentos}

Dois instrumentos foram elaborados. O primeiro é um treino cognitivo desenvolvido pelo psicólogo clínico Danyel Rocha Souza ${ }^{1}$ e será aplicado no grupo de controle. O segundo instrumento, criado pelo autor da pesquisa, é baseado em atividades selecionadas de três fontes: a tese de doutorado de Christian Puhlmann Brackmann (BRACKMANN, 2017), o teste elaborado por Marcos Román-González (ROMÁN-GONZÁLEZ, 2016) em sua tese de doutorado e o desafio Bebras (BEBRAS, 2019). Esse instrumento será executado no grupo de experimento.

Acentua-se que ambas as intervenções cognitivas possuem seis sessões e que têm como finalidade estimular a habilidade de resolução de problemas. Desta forma, busca-se, além da estimulação cognitiva, genuinamente importante ao idoso, instigar uma capacidade importante ao mercado de trabalho.

\section{Proposta de solução}

O presente estudo busca propor um conjunto de sessões de estimulação cognitiva baseadas no Pensamento Computacional, já que esse é considerado como "um processo de pensamento

${ }^{1} \mathrm{CRP} 13 / 8755$ 
VIII Congresso Brasileiro de Informática na Educação (CBIE 2019)

Anais dos Workshops do VIII Congresso Brasileiro de Informática na Educação (WCBIE 2019)

envolvido na formulação de problema e que expressa sua solução eficazmente, de tal forma que uma máquina ou pessoa possa realizar" (WING, 2006).

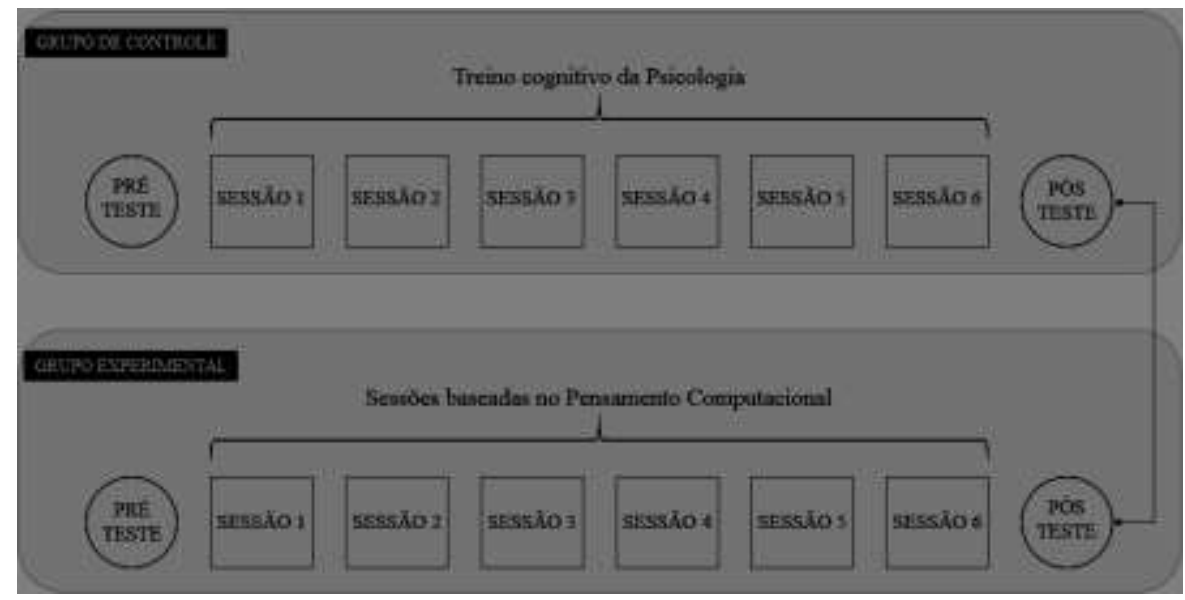

Figura 1: intervenções cognitivas a serem comparadas

Em cada grupo, antes e após a execução das sessões, será realizado o Teste de Avaliação Rápida das Funções Cognitivas - ARFC (GIL, 2005; GIL, TOULLAT \& PLUCHON, 1986) para verificar possíveis melhorias nas funções cognitivas. Como mostra a figura acima, os póstestes dos dois grupos serão comparados. Para cada sessão, em ambos os grupos, atividades serão realizadas. A Figura 2 mostra um exemplo de exercício que será praticado na sessão 2 do grupo experimental.

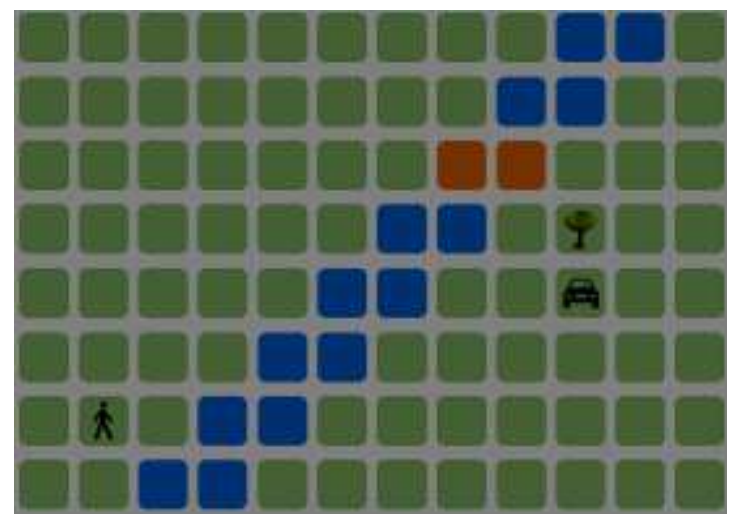

Figura 2: atividade baseada em Pensamento Computacional

O objetivo da atividade da Figura 2 é encontrar o menor caminho entre o ponto inicial (personagem) e o ponto final (automóvel) escrevendo a sequência de setas, observando algumas regras. Esse exercício estará desenvolvendo o reconhecimento de padrões e a construção de algoritmos.

\section{Resultados esperados}

Acredita-se que o resultado do pós-teste alcançado no grupo experimental será semelhante ao pós-teste do grupo de controle, possibilitando considerar as sessões de Pensamento Computacional uma alternativa de intervenção cognitiva para estimular a capacidade de resolução de problemas em idosos, com a particularidade de favorecer o aprendizado de conceitos da Computação.

O Pensamento Computacional é visto também como uma estratégia viável para o ensino de programação (ZANETTI; BORGES; RICARTE, 2016), sendo também uma nova possibilidade econômica para o público idoso.

\section{Contribuições}


Ao empreender uma pesquisa que busca associar o Pensamento Computacional com o público idoso, traz-se uma contribuição relevante, pois a maioria das iniciativas de ensino de Pensamento Computacional é voltada para os membros mais jovens da sociedade (SAUPPÉ et al., 2015). Além do mais, basear-se no Pensamento Computacional para elaborar sessões de intervenção cognitiva com o objetivo de estimular a capacidade de resolução de problemas para essa faixa etária da população é um motivo expressivo, tendo em vista que os mesmos buscam manter o cérebro estimulado e prosseguir no mercado de trabalho (GUO, 2017).

Esta pesquisa colabora também na investigação sobre os efeitos de duas intervenções cognitivas em idosos, inclusive favorecendo o aprendizado de conceitos de Computação através do Pensamento Computacional, que pode oportunizar uma progressão da atuação do idoso que deixa de ser um consumidor de tecnologia, passando a ser produtor de tecnologia através da programação como descreve Guo (2017).

\section{Referências bibliográficas}

ARGIMON, Irani I. de Lima et al. Funções executivas e a avaliação de flexibilidade de pensamento em idosos. Revista Brasileira de Ciências do Envelhecimento Humano, Passo Fundo, v. 3, n. 2, p.35-42, dez. 2006. doi:https://doi.org/10.5335/rbceh.2012.84.

BEBRAS. Bebras: International Challenge on Informatics and Computational Thinking. 2019. Disponível em: <https://www.bebras.org/>. Acesso em: 16 mar. 2019.

BRACKMANN, Christian Puhlmann. Desenvolvimento do Pensamento Computacional através de atividades desplugadas na Educação Básica. 2017. 226 f. Tese (Doutorado) Curso de Programa de Pós-graduação em Informática na Educação, Centro Interdisciplinar de Novas Tecnologias na Educação, UFRS, Porto Alegre, 2017.

CARDOSO, Nicolas de Oliveira; LANDENBERGER, Thaís; ARGIMON, Irani Iracema de Lima. Jogos Eletrônicos como Instrumentos de Intervenção no Declínio Cognitivo - Uma Revisão Sistemática. Revista de Psicologia da IMED, Passo Fundo, v. 9, n. 1, p. 119-139, nov. 2017. ISSN 2175-5027. doi:https://doi.org/10.18256/2175-5027.2017.v9i1.1941.

CAVALLI, Adriana Schüler et al. Estimulação cognitiva para melhoria das funções cerebrais de idosos. Extensio: Revista Eletrônica de Extensão, Florianópolis, v. 14, n. 27, p.101-106, 20 dez. 2017. UFSC. http://dx.doi.org/10.5007/807-0221.2017v14n27p101.

COLLINS, Anne; KOECHLIN, Etienne. Reasoning, Learning, and Creativity: Frontal Lobe Function and Human Decision-Making. Plos Biology, v. 10, n. 3, mar. 2012. Public Library of Science (PLoS). http://dx.doi.org/10.1371/journal.pbio.1001293.

GERHARDT, Tatiana Engel; SILVEIRA, Denise Tolfo. Métodos de Pesquisa. Porto Alegre: Ufrgs, 2009. 120 p. (Série Educação a Distância).

GIL, R. (2005). Neuropsicologia. (M. A. A. S, Doria, Trad.). São Paulo: SantosLiv. Ed.

GIL, R., TOULLAT, G., \& Pluchon, C. (1986). Une méthode d'evaluation rapide dês functions cognitives (ERFC). Son application à la démence senile de tupeAlzheimer. Semaine des Hôpitaux de París, 62, 2127-2133.

GUO, Philip J.. Older Adults Learning Computer Programming. Proceedings Of The 2017 Chi Conference On Human Factors In Computing Systems - Chi '17, 2017. ACM Press. http://dx.doi.org/10.1145/3025453.3025945.

IBGE - Instituto Brasileiro de Geografia e Estatística. (2018), Número de idosos cresce 18\% 
VIII Congresso Brasileiro de Informática na Educação (CBIE 2019)

Anais dos Workshops do VIII Congresso Brasileiro de Informática na Educação (WCBIE 2019)

em 5 anos e ultrapassa 30 milhões em 2017. Disponível em: 〈https://bit.ly/2xzpSK3〉. Acesso em: 09 jul. 2018.

IPEA. Idosos adiam saída do mercado de trabalho e já são 7,8\% dos trabalhadores. 2018. Disponível em: <https://bit.ly/2Y6Wrht>. Acesso em: 10 fev. 2019.

ONU. (2014), Mundo terá 2 bilhões de idosos em 2015; OMS diz que 'envelhecer bem deve ser prioridade global'. Disponível em: <https://bit.ly/2ukORjz/>. Acesso em: 01 jan. 2019.

PATI, Camila. 10 competências de que todo profissional vai precisar até 2020. 2016. Disponível em: <https://bit.ly/2ThawXu>. Acesso em: 22 jan. 2016.

ROMÁN-GONZÁLEZ, M. Codigoalfabetización y Pensamiento Computacional en Educación Primaria y Secundaria: Validación de un Instrumento y Evaluación de Programas, fev. 2016. Madrid, Spain: Universidad Nacional de Educación a Distancia.

SAUPPÉ, Allison et al. From 9 to 90. Proceedings Of The 46th Acm Technical Symposium On Computer Science Education - Sigcse '15, mar. 2015. ACM Press. http://dx.doi.org/10.1145/2676723.2677248.

SCHAIE, K. Warner; WILLIS, Sherry L.. Can decline in adult intellectual functioning be reversed? Developmental Psychology, v. 22, n. 2, p.223-232, 1986. American Psychological Association (APA). http://dx.doi.org/10.1037/0012-1649.22.2.223.

SHADISH, W. R.; COOK, T. D.; CAMPBELL, D. T. Experimental and quasi-experimental designs for generalized causal inference. 2nd ed. Boston: Houghton Mifflin, 2001.

USTÁRROZ, Javier Tirapu; CÉSPEDES, Juan Manuel Muñoz. Memoria y funciones ejecutivas. Revista de Neurología, v. 41, n. 08, p.475-484, 2005. https://doi.org/10.33588/rn.4108.2005240.

WING, J. M. Computational thinking. Communications of the ACM, v. 49, n. 3, p. 33, 2006.

ZANETTI, Humberto; BORGES, Marcos; RICARTE, Ivan. Pensamento Computacional no Ensino de Programação: Uma Revisão Sistemática da Literatura Brasileira. Anais do Xxvii Simpósio Brasileiro de Informática na Educação (sbie 2016), p.21-30, 7 nov. 2016. Sociedade Brasileira de Computação - SBC. http://dx.doi.org/10.5753/cbie.sbie.2016.21. 